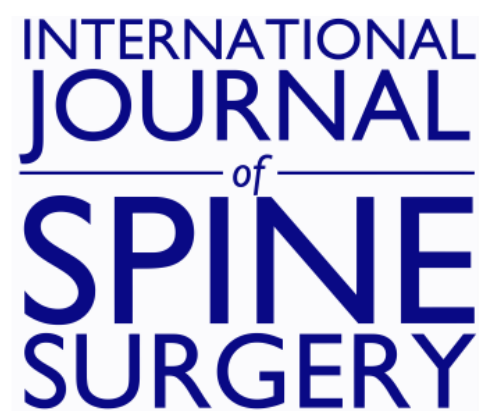

\title{
Comparative Analysis of Lumbar Spine Vertebral Morphology Between Maori and New Zealand Europeans: A Computed Tomography Study
}

William H. Cook and Joseph F. Baker

Int J Spine Surg 2021, 15 (6) 1072-1081

doi: https://doi.org/10.14444/8193

http://ijssurgery.com/content/15/6/1072

This information is current as of April 26, 2023.

Email Alerts Receive free email-alerts when new articles cite this article. Sign up at:

http://ijssurgery.com/alerts

The International Journal of Spine Surgery

2397 Waterbury Circle, Suite 1,

Aurora, IL 60504, Phone: +1-630-375-1432 


\title{
Comparative Analysis of Lumbar Spine Vertebral Morphology Between Māori and New Zealand Europeans: A Computed Tomography Study
}

\author{
WILLIAM H. COOK, MBChB AND BMedSc(Hons) ${ }^{1}$ AND JOSEPH F. BAKER, MBChB, FRCSI $^{1,2}$ \\ ${ }^{1}$ Department of Surgery, Faculty of Medical and Health Sciences, University of Auckland, Grafton, Auckland 1023, New Zealand; ${ }^{2}$ Department of Orthopaedic Surgery, \\ Waikato Hospital, Hamilton 3204, New Zealand
}

\begin{abstract}
Background: Most existing studies of lumbar anatomy do not consider ethnic influence and recruit mostly white participants. Recent studies have considered other populations; however, none have assessed Māori, the indigenous people of New Zealand (NZ). A computed tomography study of vertebral body (VB) and canal dimensions was performed for lumbar vertebrae of Māori and NZ European patients to evaluate for ethnic variation.

Methods: Lumbar vertebrae from 196 patients were measured using computed tomography. After interrater and intrarater reliability analyses, a single trained examiner measured VB heights, VB lengths, segmental angle, pedicle height and width, and vertebral canal length (VCL) and vertebral canal width for each level. Canal:body ratio was calculated. Demographic data recorded included age, sex, and ethnicity.

Results: VCL remained relatively constant through the lumbar spine; canal width increased to a maximum of $28.2 \mathrm{~mm}$ at L5. Canal:body ratios and pedicle height decreased while pedicle width increased to a maximum of $16.1 \mathrm{~mm}$ at L5. There were few differences between Māori and NZ Europeans except at the L5 level, where VCL and canal:body ratio were larger in NZ Europeans $(P<0.05)$, and pedicle height, width, and VB pediculolaminar length were larger in Māori $(P<0.05)$. Females had generally smaller measurements and age was a positive predictor of measured values $(P<0.05)$.

Conclusions: This study is the first to characterize lumbar anatomy in a Māori cohort. Adequately powered results demonstrated few differences between Māori and NZ Europeans. Isolated differences observed at L5 may be due to sacropelvic differences, which represent an area for further investigation.
\end{abstract}

Level of Evidence: 3.

Clinical Relevance: Diagnosis, surgical planning, ethnic differences.

Lumbar Spine

Keywords: lumbar vertebrae, spinal canal, tomography, x-ray computed, ethnic groups

\section{INTRODUCTION}

Spinal column anatomy varies between ethnic groups and understanding these differences is essential to ensure accurate diagnosis and appropriate treatment of spinal pathology. Existing studies of the lumbar spine demonstrate differences between black and white populations ${ }^{1}$ or define the anatomy of various Asian populations. ${ }^{2-7}$ Others have focused on defining the anatomical parameters of white or European participants, ${ }^{7}$ or do not consider ethnicity when measuring or testing spines for suitability of instrumentation. ${ }^{8-11}$

Most studies have either been conducted using cadaveric or osteological specimens or using advanced imaging modalities such as computed tomography (CT) or magnetic resonance imaging while those using cadaveric specimens have used either Vernier calipers, ${ }^{2,4,5,9,11,12}$ digital calipers, ${ }^{1,13}$ or a 3 -dimensional digitizing apparatus. ${ }^{14}$ There appears to be little difference between cadaveric and radiological modalities, ${ }^{15,16}$ and the use of advanced imaging may be more appropriate among a patient population than methodologies that utilize postmortem specimens. It is plausible that these specimens may not represent the morphometrics of the contemporary human. ${ }^{13}$

There is much interest in predicting lumbar spine stenosis using imaging modalities and previous work has aimed to define normal canal dimensions; a vertebral canal length (VCL) of less than $11.5 \mathrm{~mm}$ has often been cited as indicative of stenosis. ${ }^{17}$ However, most modern anatomic studies focus on pedicle morphology and while anatomical variations in a range of ethnicities have been elucidated, there are no published data available on spinal morphometry in New Zealand (NZ) or of a Māori population. A previous unpublished study of the cervical spine indicated some differences between 
Māori and NZ European canal:body ratios (Faraj et al, unpublished data, May 2006).

Māori are the indigenous people of NZ, comprising $17 \%$ of the population according to 2013 census data. ${ }^{18}$ By contrast, NZ European is the largest ethnic group and accounts for $70 \%$ of the NZ population, although normative values applied to the NZ population as a whole are still often based on studies performed elsewhere. $^{18}$

Understanding normal anatomic values can aid in diagnosis, treatment decisions, and surgical planning of the spine. The aim of this study was therefore to define lumbar spine morphometry in a NZ population and assess for any ethnic variation between Māori and the NZ European population.

\section{MATERIALS AND METHODS}

Local ethical body approval was obtained (HDEC: 18/CEN/8).

A trauma CT database was accessed to identify suitable imaging. All imaging was performed as part of assessment for major trauma during a 13-month period, from August 2018 to September 2019. Scans from patients older than 16 years were included if the entire spinal column, occiput to pelvis, was clearly visible and accurate vertebral body (VB) numbering could be performed. Scans were excluded if there were abnormal VB counts, transitional anatomy, evidence of spinal column disease including prior surgery, fracture, tumor, infection, or deformity, and also evidence of pelvic anomalies such as prior surgery (eg, total hip replacement), fracture, tumor, or infection that could preclude the measurement of spinal or pelvic parameters. Clinical details were not sought regarding the presence of absence of symptoms attributable to the lumbar spine.

Demographic details were collected including age, sex, and ethnicity. Only patients of Māori or NZ European ethnicities were included. All CT scans were performed with a Philips iCT (Philips, Best, The Netherlands). Axial and sagittal sequences were performed with 1 and $2 \mathrm{~mm}$ slices, respectively. IntelliSpace PACS 4.4 Enterprise (Philips, Best, The Netherlands) ruler and angle tools were used to measure each variable.

Periosteal measurements of VB anterior and posterior height; VB superior, inferior, and pediculolaminar length; segmental angle; left and right pedicle height and width; VCL; and vertebral canal width (VCW) were made at each lumbar level (Figure 1i-iii). Left and right pedicle measurements were averaged, and the mean values used for statistical analysis once statistical analysis determined absence of significant side-to-side
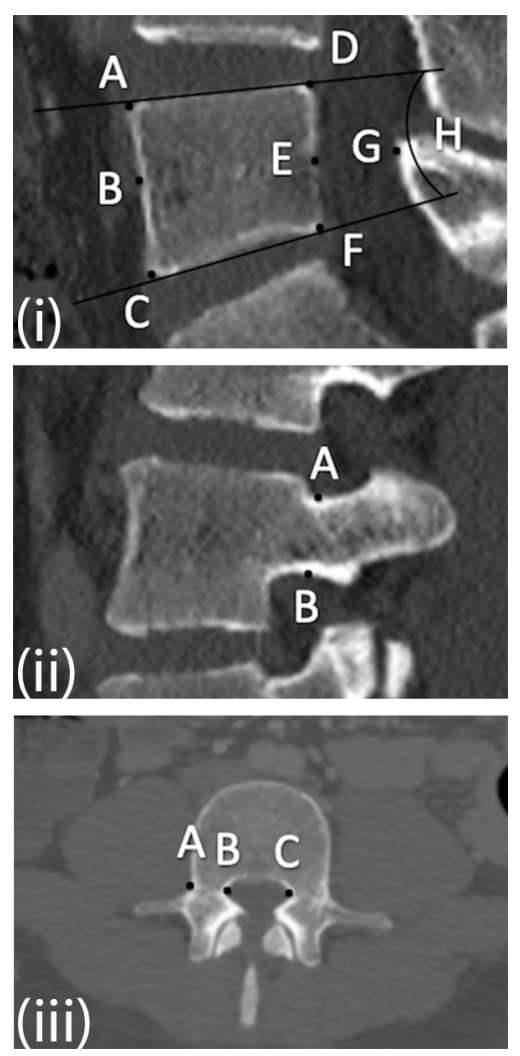

Figure 1. (i) Midsagittal computed tomography (CT) scan. Distance A-C indicates vertebral body (VB) anterior height; D-F, VB posterior height; A-D, VB superior length; B-E, VB pediculolaminar length; C-F, VB inferior length; $\mathrm{E}-\mathrm{G}$, vertebral canal length; ratio of $\mathrm{E}-\mathrm{G}$ to $\mathrm{B}-\mathrm{E}$, canal:body ratio; angle $\mathrm{H}$, segmental angle. (ii) Parasagittal CT scan. Distance A-B indicates pedicle height. (iii) Axial CT scan. Distance A-B indicates right pedicle width; B-C, vertebral canal width.

variation. Canal:body ratios were calculated by dividing VCL by VB length (Figure 1i), which was measured parallel to the VB superior endplate.

\section{Statistical Methods}

Sample size was calculated for $80 \%$ power, using a standard 5\% type I error rate, $1 \mathrm{~mm}$ effect size, and a standard deviation of $2.4 \mathrm{~mm}$ from the $\mathrm{L} 3$ canal length of a previous study. ${ }^{19}$ The calculation determined that 72 patients were required from each sex and ethnic group.

Statistical analysis was performed with Microsoft Excel 2016 (Microsoft, Redmond, WA, USA) and IBM SPSS Statistics 25 (IBM Corp, Armonk, NY, USA). Descriptive statistics were conducted for all variables at all lumbar levels. Interrater and intrarater agreement was evaluated with intraclass correlation coefficients (ICC). A washout period of 6 weeks was used before assessing intrarater reliability. Means, standard deviation, and ranges are presented where applicable. Analysis of variance was used to compare left and right pedicle heights 
Table 1. Interrater and intrarater agreement of $L 3$ radiological measurements of 10 randomly selected patients.

\begin{tabular}{lccc}
\hline Index & Mean ICC & Minimum ICC & $\begin{array}{c}\text { Maximum } \\
\text { ICC }\end{array}$ \\
\hline Interrater agreement & 0.902 & 0.782 & 0.952 \\
Intrarater agreement & 0.945 & 0.835 & 0.988 \\
\hline
\end{tabular}

Abbreviation: ICC, intraclass correlation coefficients.

and widths. Multiple linear regression was performed with age, sex, and ethnicity as independent variables and each measurement as dependent variables, while adjusting for the other 2 independent variables.

\section{RESULTS}

A total of 196 patients were included in final analysis (72 females; 75 Māori; mean age $42 \pm 19.5$ years [range, 16-98 years]).

Ten random patient $\mathrm{CT}$ scans were selected for reliability analysis with all measurements of their L3 vertebrae. A mean-measurement, absolute-agreement, 2 -way random-effects model was used. Mean interrater and intrarater ICCs were $0.902(0.782-0.952)$ and $0.945(0.835-0.988)$, respectively, for all measurements (Table 1).

Left and right pedicle heights and widths were not significantly different $(P>0.05)$ at any level and so the mean values for each level were used for subsequent analysis.

VCL remained relatively constant through the lumbar spine (Figure 2). VCW increased caudally down the lumbar spine with the narrowest mean measure at L1 of $22.4 \pm 1.8 \mathrm{~mm}$ (range, 17.4-27.0 $\mathrm{mm}$ ) and the widest at L5 with of $28.2 \pm 3.1 \mathrm{~mm}$ (range, 16.4-36.3 $\mathrm{mm}$ ) (Figure 2). Canal:body ratios decreased slightly from L1 to L5 (Figure 3). Pedicle height decreased from L1 to L5 (Figure 4). Pedicle width increased caudally with the narrowest mean measure at L1 of 7.8 $\pm 1.6 \mathrm{~mm}$ (range, $3.2-13.1 \mathrm{~mm}$ ) and the widest at L5 of $16.1 \pm 2.3 \mathrm{~mm}$ (range 11.3-24.1 mm) (Figure 4). VB measurements generally increased in size down the lumbar spine, though VB posterior height decreased (Figure 5), reflecting an increase in the segmental angle (Figure 6).

After controlling for age and sex, ethnic subgroup analysis demonstrated that NZ Europeans had longer and wider vertebral canal dimensions than Māori at all levels although these differences were only significant for VCL at L5. Canal:body ratio did not demonstrate a pattern between ethnic groups and was identical at the L1, L3, and L4 levels although was significantly larger in NZ Europeans at L5. Pedicle heights demonstrated little pattern but were significantly larger in Māori at L5. NZ Europeans had larger pedicle widths at the upper lumbar levels, which were significant at L1, but Māori had larger pedicle widths at L3, L4, and L5, with only the difference at L5 being significant.

NZ Europeans had larger VB heights than Māori, with differences significant for L1 and L2 VB anterior height and L4 VB posterior height (Tables 2-6). Conversely, VB lengths were larger in Māori with differences significant for VB superior length at all levels except L3 and for VB inferior length at all levels except L2. VB pediculolaminar length was significantly greater in Māori at only the L5 level (Tables 2-6).

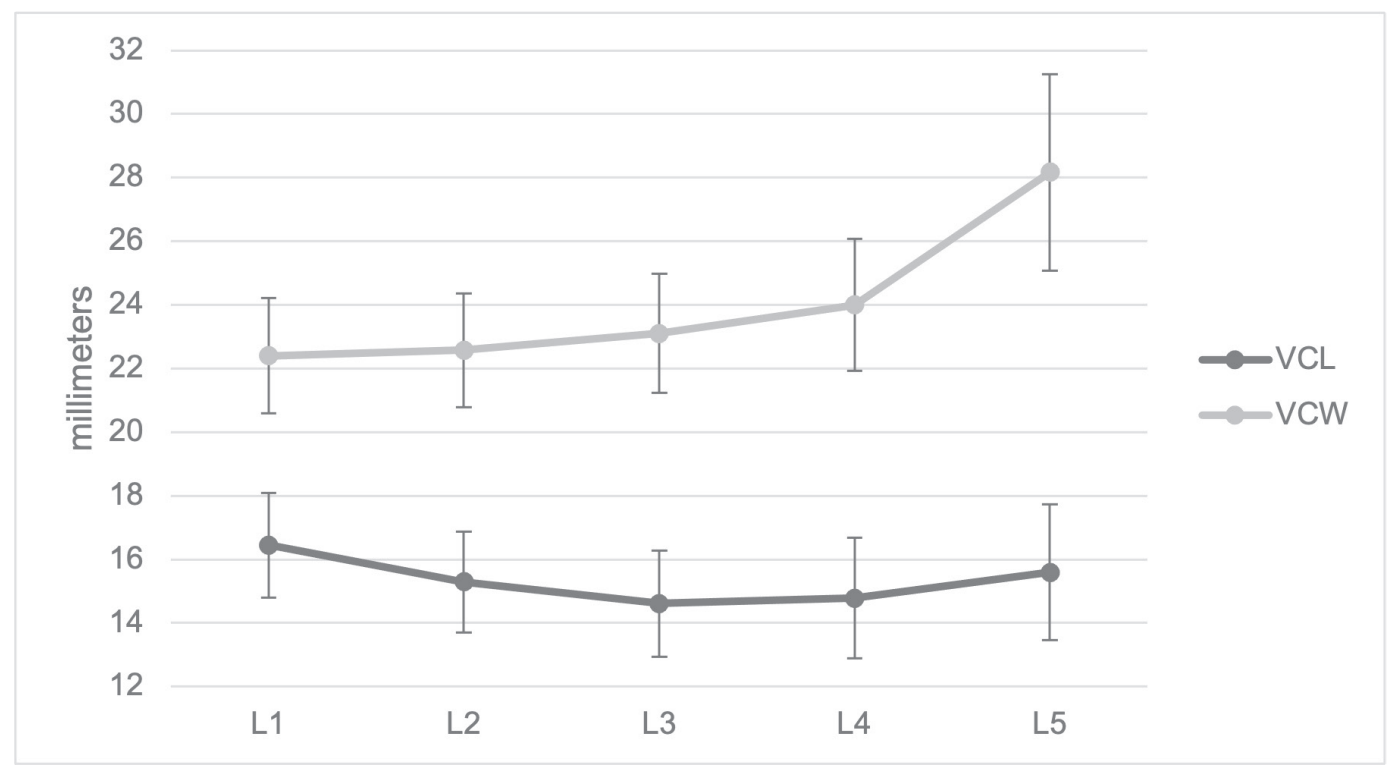

Figure 2. Lumbar vertebral canal length (VCL) and vertebral canal width (VCW). Values are mean \pm SD. 


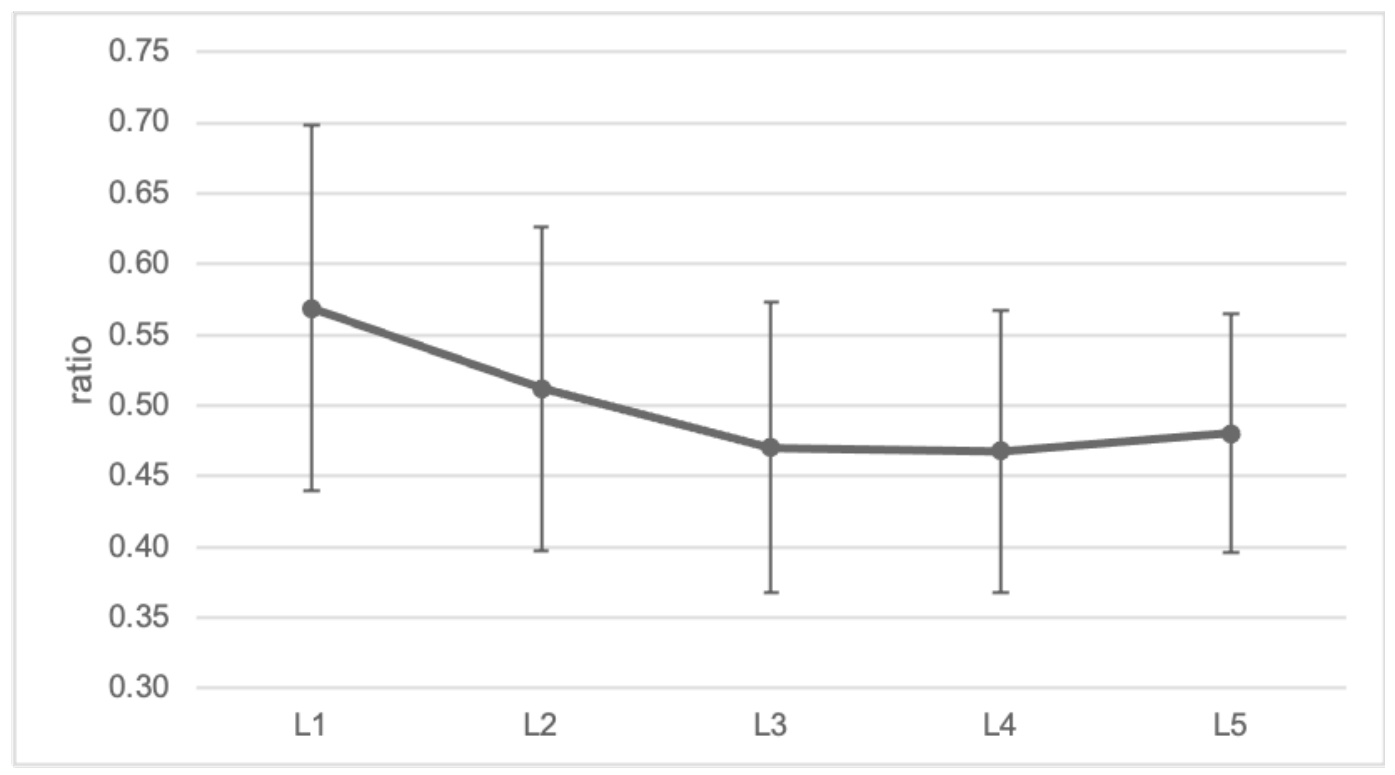

Figure 3. Lumbar canal:body ratio. Values are mean \pm SD.

After adjusting for age and ethnicity in sex subgroup analysis, females had smaller absolute measurements of all linear values except VCL at L3 and L4, which were larger by $0.1 \mathrm{~mm}$ and identical, respectively (Tables 2-6). These differences were significant at all lumbar levels for VB posterior height, VB superior, inferior, and pediculolaminar lengths, pedicle height and width, and VCW except at the L4 level where VCW was not significantly different between males and females. Females had significantly larger segmental angles and canal:body ratios at all lumbar levels except L1 where segmental angles were not significantly different.
Age was largely a positive predictor of measured values (Tables 2-6). Canal:body ratio was an exception where age was a negative predictor at all levels except L3, with significant results at L1, L2, and L5. Increasing age independently predicted a significant increase in VB superior, inferior, and pediculolaminar lengths as well as larger pedicles and VCW.

\section{DISCUSSION}

The aim of this study was to report on anatomic parameters of the lumbar spine in Māori and NZ

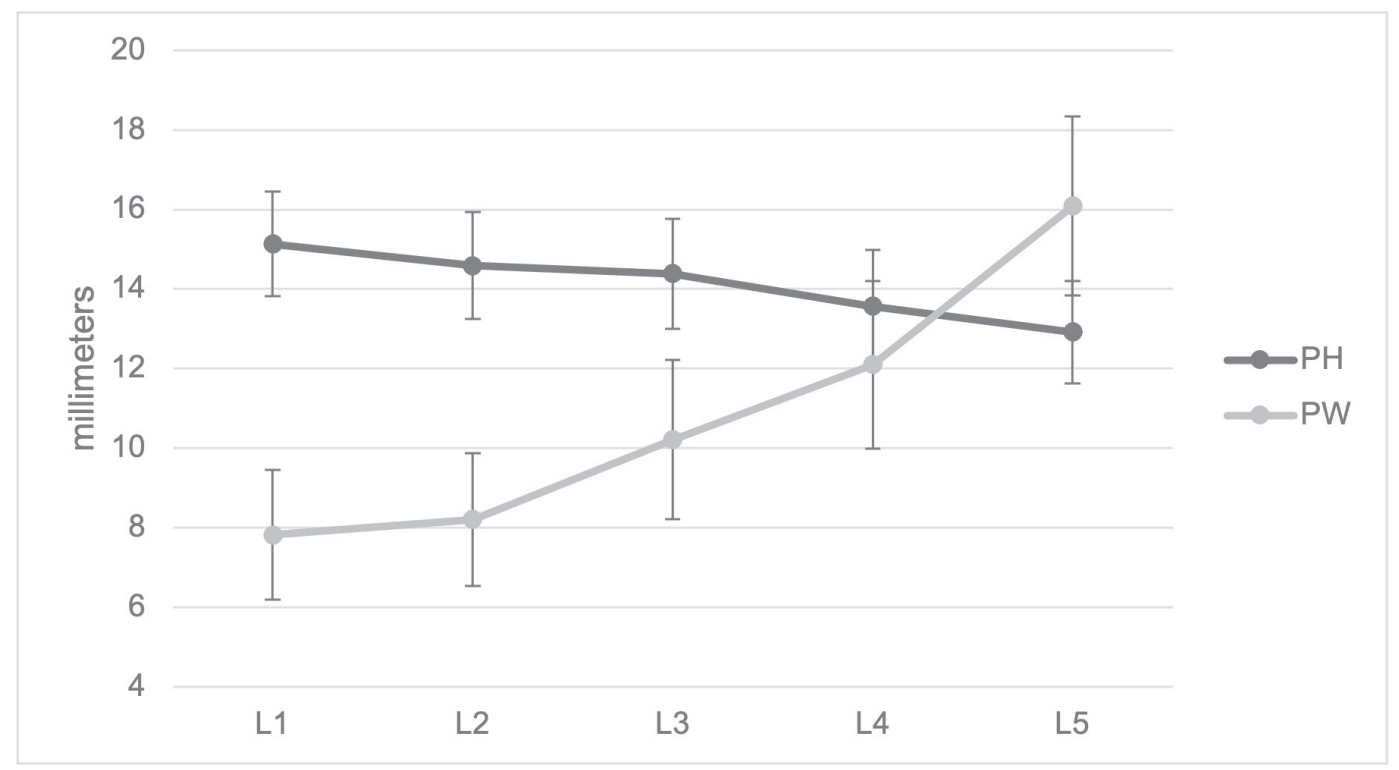

Figure 4. Lumbar pedicle height $(\mathrm{PH})$ and pedicle width $(\mathrm{PW})$. Values are mean $\pm \mathrm{SD}$. 


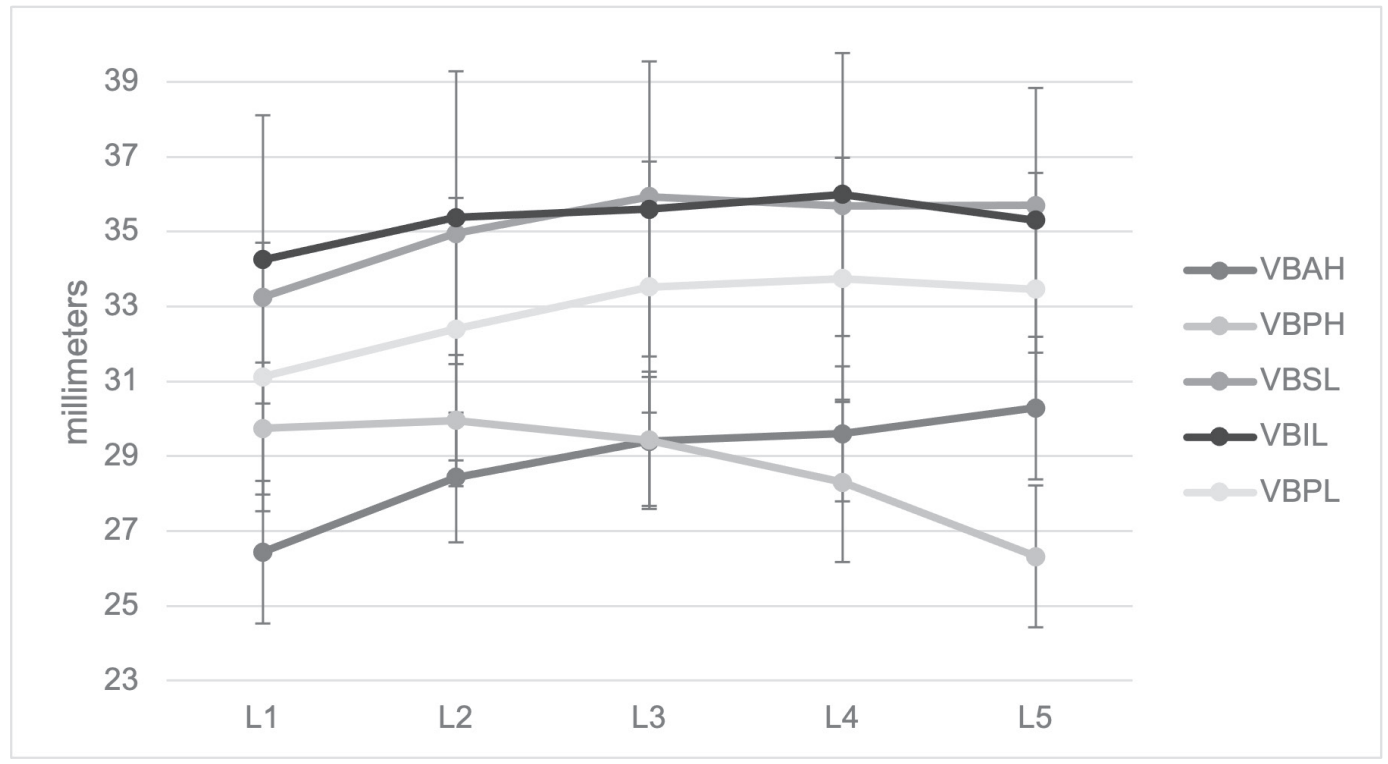

Figure 5. Lumbar vertebral body anterior height (VBAH), vertebral body posterior height (VBPH), vertebral body superior length (VBSL), vertebral body inferior length (VBIL), and vertebral body pediculolaminar length (VBPL). Values are mean \pm SD.

Europeans and test for differences in parameters between the 2 ethnic groups. Many previous studies have assessed measurable variables of the lumbar spine and both correlated these with continuous patient variables $1,3,14,20,21$ and compared measurements between sex, ${ }^{1-4,14,19-23}$ ethnicity, ${ }^{1,14}$ and disease states. ${ }^{14,22}$ Most of these focus on pedicle dimensions with few studies measuring canal dimensions. ${ }^{14,19,24}$ This is the first study to detail normative data from a NZ population and the first to provide analysis of Māori, NZ's indigenous and second largest ethnic group. ${ }^{18}$
A single previous unpublished study has compared cervical canal dimensions between Māori and NZ Europeans (Faraj et al, unpublished data, May 2006). This was in a small cohort of patients sustaining SCI and by measuring CT dimensions of their cervical spines determined that Māori had narrower VCLs than NZ Europeans by an average of $1 \mathrm{~mm}$. One may therefore hypothesize that a similar difference would exist in the lumbar spine; however, even with a suitably powered study, we did not consistently demonstrate such a difference. Faraj et al recruited patients that had sustained

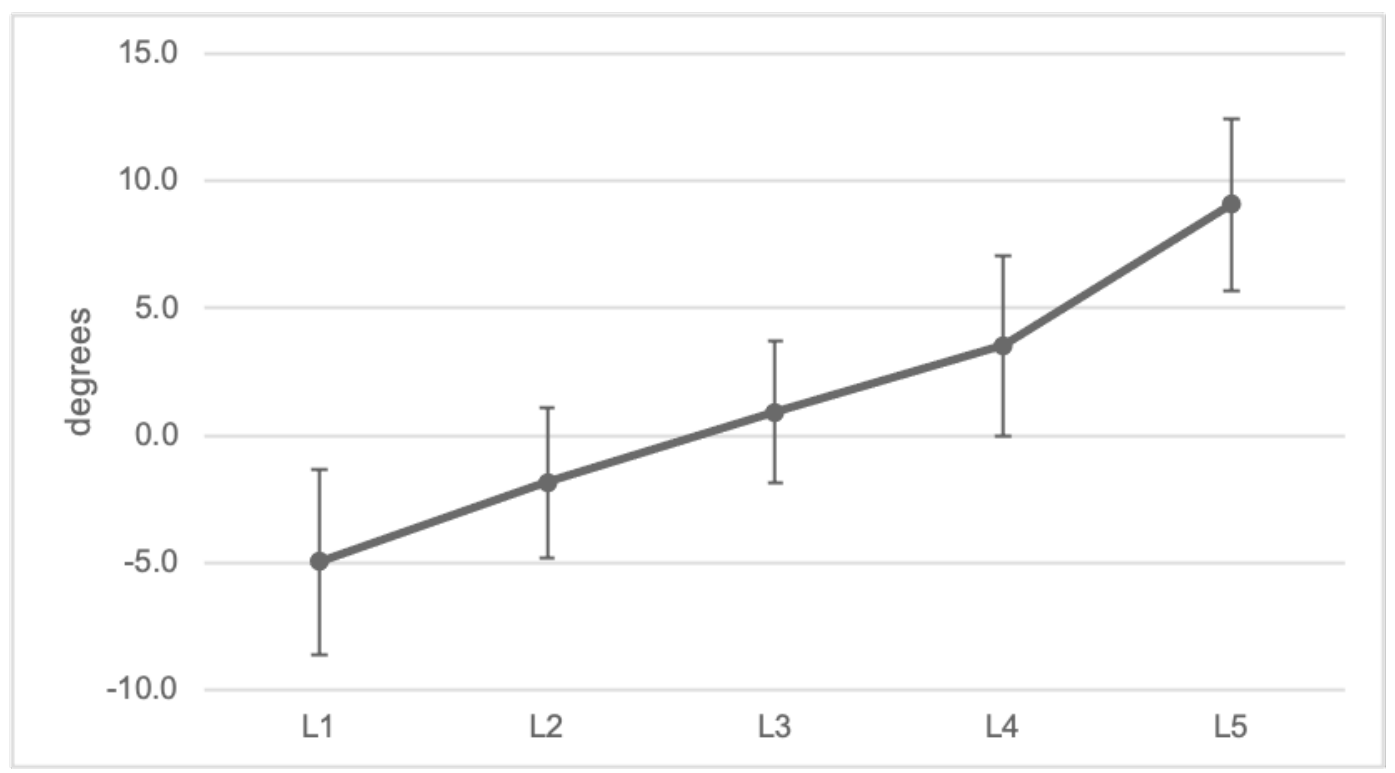

Figure 6. Lumbar segmental angle. Values are mean \pm SD. 
Table 2. Measurements (mean $\pm \mathrm{SD}$ ) at the L1 level, including comparisons of sex, ethnicity, and age.

\begin{tabular}{|c|c|c|c|c|c|c|}
\hline Demographic & VBAH (mm) & VBPH (mm) & VBSL (mm) & VBIL (mm) & VBPL (mm) & $\mathbf{S A}\left(^{\circ}\right)$ \\
\hline Total & $26.4 \pm 1.9$ & $29.7 \pm 1.8$ & $33.2 \pm 3.9$ & $34.3 \pm 4.0$ & $31.1 \pm 3.6$ & $-5.0 \pm 3.6$ \\
\hline \multicolumn{7}{|l|}{ Sex } \\
\hline Female & $26.3 \pm 2.0$ & $29.0 \pm 1.7$ & $30.7 \pm 2.7$ & $31.5 \pm 3.0$ & $28.7 \pm 2.5$ & $-4.4 \pm 3.7$ \\
\hline Male & $26.5 \pm 1.9$ & $30.2 \pm 1.6$ & $34.7 \pm 3.6$ & $35.9 \pm 3.6$ & $32.5 \pm 3.4$ & $-5.3 \pm 3.6$ \\
\hline$P$ & 0.445 & $<0.001$ & $<0.001$ & $<0.001$ & $<0.001$ & 0.127 \\
\hline \multicolumn{7}{|l|}{ Ethnicity } \\
\hline Māori & $25.9 \pm 1.7$ & $29.5 \pm 1.7$ & $33.6 \pm 4.3$ & $34.8 \pm 4.2$ & $31.4 \pm 3.6$ & $-4.9 \pm 4.4$ \\
\hline European & $26.7 \pm 2.0$ & $29.9 \pm 1.8$ & $33.0 \pm 3.6$ & $33.9 \pm 3.8$ & $31.0 \pm 3.6$ & $-5.0 \pm 3.1$ \\
\hline$P$ & 0.003 & 0.083 & 0.038 & 0.007 & 0.093 & 0.865 \\
\hline \multicolumn{7}{|l|}{ Age } \\
\hline $\operatorname{Beta}(\mathrm{mm} / \mathrm{yr})$ & -0.009 & -0.004 & 0.082 & 0.078 & 0.070 & 0.006 \\
\hline \multirow[t]{2}{*}{$P$} & 0.201 & 0.537 & $<0.001$ & $<0.001$ & $<0.001$ & 0.651 \\
\hline & PH (mm) & PW (mm) & VCL (mm) & VCW (mm) & \multicolumn{2}{|c|}{ Canal:body ratio } \\
\hline Total & $15.1 \pm 1.3$ & $7.8 \pm 1.6$ & $16.5 \pm 1.6$ & $22.4 \pm 1.8$ & \multicolumn{2}{|c|}{$0.57 \pm 0.13$} \\
\hline \multicolumn{7}{|l|}{ Sex } \\
\hline Female & $14.6 \pm 1.3$ & $6.9 \pm 1.6$ & $16.3 \pm 1.3$ & $21.8 \pm 1.9$ & \multicolumn{2}{|c|}{$0.63 \pm 0.11$} \\
\hline Male & $15.5 \pm 1.2$ & $8.3 \pm 1.4$ & $16.5 \pm 1.8$ & $22.7 \pm 1.7$ & \multicolumn{2}{|c|}{$0.53 \pm 0.13$} \\
\hline$P$ & $<0.001$ & $<0.001$ & 0.265 & $<0.001$ & \multicolumn{2}{|c|}{$<0.001$} \\
\hline \multicolumn{7}{|l|}{ Ethnicity } \\
\hline Māori & $15.0 \pm 1.2$ & $7.5 \pm 1.5$ & $16.2 \pm 1.4$ & $22.1 \pm 1.4$ & \multicolumn{2}{|c|}{$0.57 \pm 0.11$} \\
\hline European & $15.2 \pm 1.4$ & $8.0 \pm 1.7$ & $16.6 \pm 1.8$ & $22.6 \pm 2.0$ & \multicolumn{2}{|c|}{$0.57 \pm 0.14$} \\
\hline$P$ & 0.183 & 0.012 & 0.108 & 0.162 & \multicolumn{2}{|c|}{0.611} \\
\hline \multicolumn{7}{|l|}{ Age } \\
\hline $\operatorname{Beta}(\mathrm{mm} / \mathrm{y})$ & 0.008 & 0.016 & -0.002 & 0.019 & \multicolumn{2}{|c|}{-0.001} \\
\hline$P$ & 0.082 & 0.004 & 0.736 & 0.003 & \multicolumn{2}{|c|}{0.013} \\
\hline
\end{tabular}

Abbreviations: PH, pedicle height; PW, pedicle width; SA, segmental angle; VBAH, vertebral body anterior height; VBIL, vertebral body inferior length; VBPH, vertebral body posterior height; VBPL, vertebral body pediculolaminar length; VBSL, vertebral body superior length; VCL, vertebral canal length; VCW, vertebral canal width. Note: Canal:body ratio, ratio of canal length to vertebral body length at the pediculolaminar level.

Table 3. Measurements (mean \pm SD) at the L2 level, including comparisons of sex, ethnicity, and age.

\begin{tabular}{|c|c|c|c|c|c|c|}
\hline Demographic & VBAH (mm) & VBPH (mm) & VBSL (mm) & VBIL (mm) & VBPL (mm) & SA $\left(^{\circ}\right)$ \\
\hline Total & $28.4 \pm 1.7$ & $30.0 \pm 1.8$ & $35.0 \pm 3.9$ & $35.4 \pm 3.8$ & $32.4 \pm 3.5$ & $-1.8 \pm 3.0$ \\
\hline \multicolumn{7}{|l|}{ Sex } \\
\hline Female & $28.4 \pm 1.8$ & $29.3 \pm 1.8$ & $32.1 \pm 2.5$ & $32.7 \pm 2.6$ & $30.2 \pm 2.4$ & $-1.1 \pm 2.9$ \\
\hline Male & $28.5 \pm 1.7$ & $30.3 \pm 1.7$ & $36.6 \pm 3.7$ & $36.9 \pm 3.6$ & $33.7 \pm 3.4$ & $-2.3 \pm 2.9$ \\
\hline$P$ & 0.620 & $<0.001$ & $<0.001$ & $<0.001$ & $<0.001$ & 0.007 \\
\hline \multicolumn{7}{|l|}{ Ethnicity } \\
\hline Māori & $28.1 \pm 1.7$ & $29.7 \pm 1.7$ & $35.5 \pm 4.1$ & $35.7 \pm 3.8$ & $32.5 \pm 3.8$ & $-2.0 \pm 2.3$ \\
\hline European & $28.6 \pm 1.7$ & $30.1 \pm 1.8$ & $34.6 \pm 3.8$ & $35.2 \pm 3.8$ & $32.3 \pm 3.3$ & $-1.7 \pm 3.3$ \\
\hline$P$ & 0.035 & 0.052 & 0.010 & 0.053 & 0.231 & 0.705 \\
\hline \multicolumn{7}{|l|}{ Age } \\
\hline Beta $(\mathrm{mm} / \mathrm{yr})$ & 0.004 & -0.002 & 0.076 & 0.075 & 0.067 & 0.022 \\
\hline \multirow[t]{2}{*}{$P$} & 0.516 & 0.719 & $<0.001$ & $<0.001$ & $<0.001$ & 0.041 \\
\hline & PH (mm) & PW (mm) & VCL (mm) & VCW (mm) & \multicolumn{2}{|c|}{ Canal:body ratio } \\
\hline Total & $14.6 \pm 1.3$ & $8.2 \pm 1.7$ & $15.3 \pm 1.6$ & $22.6 \pm 1.8$ & \multicolumn{2}{|c|}{$0.51 \pm 0.12$} \\
\hline \multicolumn{7}{|l|}{ Sex } \\
\hline Female & $14.1 \pm 1.2$ & $7.3 \pm 1.5$ & $15.2 \pm 1.4$ & $22.0 \pm 1.8$ & \multicolumn{2}{|c|}{$0.57 \pm 0.12$} \\
\hline Male & $14.9 \pm 1.3$ & $8.7 \pm 1.5$ & $15.4 \pm 1.7$ & $22.9 \pm 1.7$ & \multicolumn{2}{|c|}{$0.48 \pm 0.10$} \\
\hline$P$ & $<0.001$ & $<0.001$ & 0.392 & $<0.001$ & \multicolumn{2}{|c|}{$<0.001$} \\
\hline \multicolumn{7}{|l|}{ Ethnicity } \\
\hline Māori & $14.6 \pm 1.2$ & $8.1 \pm 1.7$ & $15.0 \pm 1.4$ & $22.2 \pm 1.6$ & \multicolumn{2}{|c|}{$0.52 \pm 0.13$} \\
\hline European & $14.6 \pm 1.4$ & $8.3 \pm 1.7$ & $15.4 \pm 1.7$ & $22.8 \pm 1.9$ & \multicolumn{2}{|c|}{$0.51 \pm 0.10$} \\
\hline$P$ & 0.891 & 0.670 & 0.116 & 0.058 & \multicolumn{2}{|c|}{0.663} \\
\hline \multicolumn{7}{|l|}{ Age } \\
\hline $\operatorname{Beta}(\mathrm{mm} / \mathrm{y})$ & -0.005 & 0.017 & 0.004 & 0.023 & \multicolumn{2}{|c|}{-0.001} \\
\hline$P$ & 0.338 & 0.003 & 0.488 & $<0.001$ & \multicolumn{2}{|c|}{0.041} \\
\hline
\end{tabular}

Abbreviations: PH, pedicle height; PW, pedicle width; SA, segmental angle; VBAH, vertebral body anterior height; VBIL, vertebral body inferior length; VBPH, vertebral body posterior height; VBPL, vertebral body pediculolaminar length; VBSL, vertebral body superior length; VCL, vertebral canal length; VCW, vertebral canal width. Note: Canal:body ratio, ratio of canal length to vertebral body length at the pediculolaminar level. 
Table 4. Measurements (mean \pm SD) at the L3 level, including comparisons of sex, ethnicity, and age.

\begin{tabular}{|c|c|c|c|c|c|c|}
\hline Demographic & VBAH (mm) & VBPH (mm) & VBSL (mm) & VBIL (mm) & VBPL (mm) & SA $\left(^{\circ}\right)$ \\
\hline Total & $29.4 \pm 1.7$ & $29.4 \pm 1.8$ & $35.9 \pm 4.0$ & $35.6 \pm 3.8$ & $33.5 \pm 3.4$ & $0.9 \pm 2.8$ \\
\hline \multicolumn{7}{|l|}{ Sex } \\
\hline Female & $29.1 \pm 2.0$ & $28.8 \pm 1.8$ & $33.2 \pm 2.6$ & $32.8 \pm 2.6$ & $31.1 \pm 2.3$ & $1.8 \pm 3.1$ \\
\hline Male & $29.6 \pm 1.6$ & $29.8 \pm 1.8$ & $37.5 \pm 3.7$ & $37.2 \pm 3.5$ & $34.9 \pm 3.1$ & $0.4 \pm 2.5$ \\
\hline$P$ & 0.081 & $<0.001$ & $<0.001$ & $<0.001$ & $<0.001$ & 0.002 \\
\hline \multicolumn{7}{|l|}{ Ethnicity } \\
\hline Māori & $29.1 \pm 1.6$ & $29.1 \pm 1.7$ & $36.2 \pm 3.8$ & $36.0 \pm 3.9$ & $33.7 \pm 3.2$ & $0.9 \pm 2.6$ \\
\hline European & $29.6 \pm 1.8$ & $29.6 \pm 1.9$ & $35.8 \pm 4.1$ & $35.4 \pm 3.8$ & $33.4 \pm 2.9$ & $1.0 \pm 2.9$ \\
\hline$P$ & 0.080 & 0.084 & 0.064 & 0.019 & 0.179 & 0.988 \\
\hline \multicolumn{7}{|l|}{ Age } \\
\hline $\operatorname{Beta}(\mathrm{mm} / \mathrm{yr})$ & 0.002 & 0.002 & 0.084 & 0.087 & 0.066 & 0.019 \\
\hline \multirow[t]{2}{*}{$P$} & 0.707 & 0.768 & $<0.001$ & $<0.001$ & $<0.001$ & 0.055 \\
\hline & PH (mm) & PW (mm) & VCL (mm) & VCW (mm) & \multicolumn{2}{|c|}{ Canal:body ratio } \\
\hline Total & $14.4 \pm 1.4$ & $10.2 \pm 2.0$ & $14.6 \pm 1.7$ & $23.1 \pm 1.9$ & 0.47 & \\
\hline \multicolumn{7}{|l|}{ Sex } \\
\hline Female & $13.9 \pm 1.2$ & $9.2 \pm 1.8$ & $14.7 \pm 1.4$ & $22.6 \pm 1.7$ & \multicolumn{2}{|c|}{$0.53 \pm 0.11$} \\
\hline Male & $14.7 \pm 0.4$ & $10.8 \pm 1.9$ & $14.6 \pm 1.8$ & $23.4 \pm 1.9$ & \multicolumn{2}{|c|}{$0.44 \pm 0.08$} \\
\hline$P$ & $<0.001$ & $<0.001$ & 0.703 & 0.002 & \multicolumn{2}{|c|}{$<0.001$} \\
\hline \multicolumn{7}{|l|}{ Ethnicity } \\
\hline Māori & $14.4 \pm 1.3$ & $10.4 \pm 1.9$ & $14.4 \pm 1.3$ & $22.8 \pm 1.6$ & \multicolumn{2}{|c|}{$0.47 \pm 0.11$} \\
\hline European & $14.4 \pm 1.5$ & $10.1 \pm 2.1$ & $14.8 \pm 1.9$ & $23.3 \pm 2.0$ & \multicolumn{2}{|c|}{$0.47 \pm 0.10$} \\
\hline$P$ & 0.709 & 0.147 & 0.195 & 0.088 & \multicolumn{2}{|c|}{0.835} \\
\hline \multicolumn{7}{|l|}{ Age } \\
\hline $\operatorname{Beta}(\mathrm{mm} / \mathrm{y})$ & 0.001 & 0.016 & 0.010 & 0.023 & \multicolumn{2}{|c|}{0.000} \\
\hline$P$ & 0.895 & 0.021 & 0.117 & 0.001 & \multicolumn{2}{|c|}{0.197} \\
\hline
\end{tabular}

Abbreviations: PH, pedicle height; PW, pedicle width; SA, segmental angle; VBAH, vertebral body posterior height; VBAH, vertebral body anterior height; VBIL, vertebral body inferior length; VBPL, vertebral body pediculolaminar length; VBSL, vertebral body superior length; VCL, vertebral canal length; VCW, vertebral canal width.

Note: Canal:body ratio, ratio of canal length to vertebral body length at the pediculolaminar level.

Table 5. Measurements (mean \pm SD) at the L4 level, including comparisons of sex, ethnicity, and age.

\begin{tabular}{|c|c|c|c|c|c|c|}
\hline Demographic & VBAH (mm) & VBPH (mm) & VBSL (mm) & VBIL (mm) & VBPL (mm) & SA $\left(^{\circ}\right)$ \\
\hline Total & $29.6 \pm 1.8$ & $28.3 \pm 2.1$ & $35.7 \pm 3.8$ & $36.0 \pm 3.7$ & $33.7 \pm 3.2$ & $3.6 \pm 3.6$ \\
\hline \multicolumn{7}{|l|}{ Sex } \\
\hline Female & $29.3 \pm 1.8$ & $27.2 \pm 2.0$ & $33.1 \pm 2.7$ & $33.3 \pm 2.8$ & $31.4 \pm 2.4$ & $5.1 \pm 3.6$ \\
\hline Male & $29.8 \pm 1.8$ & $28.9 \pm 2.0$ & $37.2 \pm 3.5$ & $37.5 \pm 3.3$ & $35.1 \pm 2.8$ & $2.6 \pm 3.2$ \\
\hline$P$ & 0.071 & $<0.001$ & $<0.001$ & $<0.001$ & $<0.001$ & $<0.001$ \\
\hline \multicolumn{7}{|l|}{ Ethnicity } \\
\hline Māori & $29.3 \pm 1.9$ & $27.7 \pm 2.0$ & $36.1 \pm 4.0$ & $36.3 \pm 3.6$ & $34.0 \pm 3.2$ & $4.1 \pm 3.3$ \\
\hline European & $29.8 \pm 1.8$ & $28.7 \pm 2.2$ & $35.4 \pm 3.7$ & $35.8 \pm 3.8$ & $33.6 \pm 3.2$ & $3.2 \pm 3.6$ \\
\hline$P$ & 0.075 & 0.002 & 0.011 & 0.037 & 0.079 & 0.052 \\
\hline \multicolumn{7}{|l|}{ Age } \\
\hline $\operatorname{Beta}(\mathrm{mm} / \mathrm{yr})$ & -0.006 & 0.008 & 0.086 & 0.081 & 0.064 & 0.000 \\
\hline \multirow[t]{2}{*}{$P$} & 0.402 & 0.263 & $<0.001$ & $<0.001$ & $<0.001$ & 0.970 \\
\hline & PH (mm) & PW (mm) & VCL (mm) & VCW (mm) & \multicolumn{2}{|c|}{ Canal:body ratio } \\
\hline Total & $13.6 \pm 1.4$ & $12.1 \pm 2.1$ & $14.8 \pm 1.9$ & $24.0 \pm 2.1$ & 0.47 & \\
\hline \multicolumn{7}{|l|}{ Sex } \\
\hline Female & $12.9 \pm 1.3$ & $11.2 \pm 2.2$ & $14.8 \pm 1.6$ & $23.7 \pm 1.9$ & \multicolumn{2}{|c|}{$0.52 \pm 0.10$} \\
\hline Male & $14.0 \pm 1.3$ & $12.6 \pm 1.9$ & $14.8 \pm 1.6$ & $24.1 \pm 2.2$ & \multicolumn{2}{|c|}{$0.44 \pm 0.08$} \\
\hline$P$ & $<0.001$ & $<0.001$ & 0.933 & 0.148 & \multicolumn{2}{|c|}{$<0.001$} \\
\hline \multicolumn{7}{|l|}{ Ethnicity } \\
\hline Māori & $13.6 \pm 1.4$ & $12.3 \pm 2.4$ & $14.6 \pm 1.9$ & $23.9 \pm 1.9$ & \multicolumn{2}{|c|}{$0.47 \pm 0.11$} \\
\hline European & $13.6 \pm 1.5$ & $11.9 \pm 1.9$ & $14.9 \pm 1.9$ & $24.1 \pm 2.2$ & \multicolumn{2}{|c|}{$0.47 \pm 0.09$} \\
\hline$P$ & 0.987 & 0.114 & 0.456 & 0.672 & \multicolumn{2}{|c|}{0.773} \\
\hline \multicolumn{7}{|l|}{ Age } \\
\hline $\operatorname{Beta}(\mathrm{mm} / \mathrm{y})$ & 0.007 & 0.015 & 0.008 & 0.024 & \multicolumn{2}{|c|}{-0.001} \\
\hline$P$ & 0.147 & 0.048 & 0.281 & 0.002 & \multicolumn{2}{|c|}{0.116} \\
\hline
\end{tabular}

Abbreviations: PH, pedicle height; PW, pedicle width; SA, segmental angle; VBAH, vertebral body anterior height; VBIL, vertebral body inferior length; VBPH, vertebral body posterior height; VBPL, vertebral body pediculolaminar length; VBSL, vertebral body superior length; VCL, vertebral canal length; VCW, vertebral canal width. Note: Canal:body ratio, ratio of canal length to vertebral body length at the pediculolaminar level. 
Table 6. Measurements (mean \pm SD) at the L5 level, including comparisons of sex, ethnicity, and age.

\begin{tabular}{|c|c|c|c|c|c|c|}
\hline Demographic & VBAH (mm) & VBPH (mm) & VBSL (mm) & VBIL (mm) & VBPL (mm) & SA $\left(^{\circ}\right)$ \\
\hline Total & $30.3 \pm 1.9$ & $26.3 \pm 1.9$ & $35.7 \pm 3.5$ & $35.3 \pm 3.6$ & $33.5 \pm 3.1$ & $3.6 \pm 3.6$ \\
\hline \multicolumn{7}{|l|}{ Sex } \\
\hline Female & $29.6 \pm 1.8$ & $25.5 \pm 1.6$ & $33.3 \pm 2.6$ & $32.8 \pm 3.0$ & $31.4 \pm 2.6$ & $5.1 \pm 3.6$ \\
\hline Male & $30.7 \pm 1.9$ & $26.8 \pm 1.9$ & $37.1 \pm 3.2$ & $36.7 \pm 3.1$ & $34.6 \pm 2.8$ & $2.6 \pm 3.2$ \\
\hline$P$ & $<0.001$ & $<0.001$ & $<0.001$ & $<0.001$ & $<0.001$ & 0.013 \\
\hline \multicolumn{7}{|l|}{ Ethnicity } \\
\hline Māori & $30.1 \pm 2.0$ & $26.1 \pm 1.7$ & $36.4 \pm 3.4$ & $35.9 \pm 3.6$ & $33.8 \pm 3.1$ & $8.9 \pm 3.2$ \\
\hline European & $30.4 \pm 1.8$ & $26.4 \pm 2.0$ & $35.3 \pm 3.5$ & $35.0 \pm 3.6$ & $33.2 \pm 3.1$ & $9.2 \pm 3.5$ \\
\hline$P$ & 0.343 & 0.293 & $<0.001$ & 0.003 & 0.019 & 0.511 \\
\hline \multicolumn{7}{|l|}{ Age } \\
\hline $\operatorname{Beta}(\mathrm{mm} / \mathrm{y})$ & -0.003 & 0.001 & 0.084 & 0.071 & 0.061 & 0.002 \\
\hline \multirow[t]{2}{*}{$P$} & 0.620 & 0.922 & $<0.001$ & $<0.001$ & $<0.001$ & 0.890 \\
\hline & PH (mm) & PW (mm) & VCL (mm) & VCW (mm) & \multicolumn{2}{|c|}{ Canal:body ratio } \\
\hline Total & $12.9 \pm 1.9$ & $16.1 \pm 2.3$ & $15.6 \pm 2.1$ & $28.2 \pm 3.1$ & \multicolumn{2}{|c|}{$0.48 \pm 0.08$} \\
\hline \multicolumn{7}{|l|}{ Sex } \\
\hline Female & $12.5 \pm 1.0$ & $15.1 \pm 1.8$ & $15.1 \pm 1.9$ & $27.6 \pm 2.9$ & \multicolumn{2}{|c|}{$0.50 \pm 0.09$} \\
\hline Male & $13.1 \pm 1.4$ & $16.6 \pm 2.3$ & $15.9 \pm 2.2$ & $28.5 \pm 3.2$ & \multicolumn{2}{|c|}{$0.47 \pm 0.08$} \\
\hline$P$ & $<0.001$ & $<0.001$ & 0.010 & 0.033 & \multicolumn{2}{|c|}{0.009} \\
\hline \multicolumn{7}{|l|}{ Ethnicity } \\
\hline Māori & $13.3 \pm 1.2$ & $16.5 \pm 2.3$ & $15.1 \pm 1.8$ & $28.1 \pm 3.1$ & \multicolumn{2}{|c|}{$0.47 \pm 0.08$} \\
\hline European & $12.7 \pm 1.3$ & $15.8 \pm 2.2$ & $15.9 \pm 2.3$ & $28.2 \pm 3.1$ & \multicolumn{2}{|c|}{$0.49 \pm 0.09$} \\
\hline$P$ & $<0.001$ & 0.013 & 0.018 & 0.997 & \multicolumn{2}{|c|}{0.028} \\
\hline \multicolumn{7}{|l|}{ Age } \\
\hline $\operatorname{Beta}(\mathrm{mm} / \mathrm{y})$ & 0.011 & 0.033 & 0.006 & 0.033 & \multicolumn{2}{|c|}{-0.001} \\
\hline$P$ & 0.013 & $<0.001$ & 0.471 & 0.004 & \multicolumn{2}{|c|}{0.027} \\
\hline
\end{tabular}

Abbreviations: PH, pedicle height; PW, pedicle width; SA, segmental angle; VBAH, vertebral body (VB) anterior height; VBIL, VB inferior length; VBPH, VB posterior height; VBSL, VB superior length; VCL, vertebral canal length; VCW, vertebral canal width.

Note: Canal:body ratio, ratio of canal length to vertebral body length at the pediculolaminar level.

SCI and so a selection bias may have influenced their findings; however, our recruitment was irrespective of any previous spinal complaints and the presence of current symptoms was not determined.

In the present study, there was no evidence for a difference in vertebral canal dimensions between the 2 ethnic groups except at L5 where Māori demonstrated a slightly shorter VCL. The mean L5 VCL was similar to other normal populations. ${ }^{14,19}$ Karantanas et al (1998) noted that their measurements of VCL demonstrated no significant correlation with age, height, or weight but did not record and assess sex and ethnicity as confounding variables. ${ }^{19}$ We hypothesize differences at L5 may reflect differences in sacropelvic anatomy between the 2 ethnic groups as the L5 represents a transition from the large, rigid pelvic "base" to the mobile spinal column.

Although the difference at L5 was not very large, it is worth noting that most stenosis occurs at the L4-L5 level, for both congenital and acquired types. ${ }^{25,26}$ The canal:body ratio was first developed to predict cervical spinal stenosis, ${ }^{27}$ and more recently assessed as a tool for detecting lumbar spinal stenosis. ${ }^{13}$ Understanding the normative values for a population is essential when attempting to determine whether an individual may have congenital narrowing of the spinal canal and therefore be predisposed to development of claudicant or radiculopathic symptoms. Not only may this influence surgical decision-making, but preexisting conditions may influence insurance cover in certain health systems. ${ }^{28}$

Previous work by Bajwa et al has proposed using a ratio of $<0.5$ to predict stenosis, ${ }^{13}$ however, the mean ratio in the present study was $<0.5$ at the lower lumbar levels. So, this appears likely to "over diagnose" the presence of stenosis. An accepted ratio or absolute measure to define stenosis within the lumbar spine remains elusive. Although the design of the present study precluded us from correlating our measurements with clinical history, it is unlikely that more people would have had symptoms. Such ratios should therefore be used cautiously without available supplementary clinical information and indeed the use of such ratios does not have universal support in the literature. ${ }^{29}$ Linear measurements of the canal have also been found to correlate poorly with clinical symptoms of spinal stenosis. $^{24}$

Pedicle dimensions in the present study were significantly larger in Māori at the L5 level only. Studies including specimens or imaging from multiple ethnic groups have demonstrated few differences between them, ${ }^{12}$ or differences that do not follow a particular pattern. ${ }^{1}$ Such studies of specific ethnic groups constitute a large proportion of the literature on pedicle morphology and authors often offer conclusions after 
comparing their mean results with other series. ${ }^{2-7} \mathrm{We}$ are reassured that with similar dimensions of pedicle anatomy between the 2 ethnic groups that surgical planning need not differ significantly greatly when considering size of pedicle screws.

Body morphometrics including height and weight were also not assessed and have been found to correlate with vertebral measurements elsewhere. ${ }^{1,19}$ Body morphometrics are inconsistently recorded in medical records and in the unwell individual are possibly skewed, for example, due to either blood loss in the setting of major trauma or fluid resuscitation. Nonetheless, these values would be desirable in future studies.

\section{CONCLUSION}

The present study has been the first to characterize the lumbar anatomy of a Māori population and compare it to a local NZ European population. After adjusting for age and sex, isolated differences in vertebral canal and pedicle dimensions at the L5 level were observed while the majority of measures were not significantly different. Further evaluation of sacropelvic anatomy is warranted to understand the differences observed at L5. As there are no obvious major differences between Māori and NZ European lumbar anatomy, we can safely apply wider normative population values to both ethnic groups without fear of inappropriate application.

\section{ACKNOWLEDGMENTS}

W.H.C. was the recipient of a NuVasive ${ }^{\circledR}$ studentship grant and would like to thank the support of the Waikato Clinical Campus.

\section{REFERENCES}

1. Yu CC, Yuh RT, Bajwa NS, Toy JO, Ahn UM, Ahn NU. Pedicle morphometry of lumbar veMorphometry of lumbar vertebrae. Spine. 2015;40(21):1639-1646. doi:10.1097/BRS.0000000000001086

2. Kim NH, Lee HM, Chung IH, Kim HJ, Kim SJ. Morphometric study of the pedicles of thoracic and lumbar vertebrae in Koreans. Spine. 1994;19(12):1390-1394. doi:10.1097/00007632199406000-00014

3. Cheung KMC, Ruan D, Chan FL, Fang D. Computed tomographic osteometry of asian lumbar pedicles. Spine. 1994;19(13):1495-1498. doi:10.1097/00007632-199407000-00014

4. Mitra SR, Datir SP, Jadhav SO. Morphometric study of the lumbar pedicle in the indian population as related to pedicular screw fixation. Spine. 2002;27(5):453-459. doi:10.1097/00007632200203010-00004

5. Hou S, Hu R, Shi Y. Pedicle morphology of the lower thoracic and lumbar spine in a Chinese population. Spine. 1993;18(13):1850-1855. doi:10.1097/00007632-199310000-00021
6. Acharya S, Dorje T, Srivastava A. Lower dorsal and lumbar pedicle morphometry in indian population: A study of four hundred fifty vertebrae. Spine. 2010;35(10):E378-84. doi:10.1097/ BRS.0b013e3181cb7f2b

7. Chadha M, Balain B, Maini L, Dhaon BK. Pedicle morphology of the lower thoracic, lumbar, and s1 vertebrae: an indian perspective. Spine. 2003;28(8):744-749. doi:10.1097/01. BRS.0000058929.92839.1A

8. Karakas P, Gulhal Bozkir M. Lumbar vertebrae: anatomic and radiologic perspectives related to surgical procedures. $\mathrm{Neu}$ rosurgery Quarterly. 2006;16(2):81-84. doi:10.1097/00013414200606000-00006

9. Olsewski JM, Simmons EH, Kallen FC, Mendel FC, Severin CM, Berens DL. Morphometry of the lumbar spine: anatomical perspectives related to transpedicular fixation. J Bone Jt Surg Am. 1990;72(A4):541-549. doi:10.2106/00004623-199072040-00011

10. Zindrick MR, Wiltse LL, Widell EH, et al. A biomechanical study of intrapeduncular screw fixation in the lumbosacral spine. Clin Orthop Relat Res. 1986;203:99. doi:10.1097/00003086198602000-00012

11. Berry JL, Moran JM, Berg WS, Steffee AD. A morphometric study of human lumbar and selected thoracic vertebrae. Spine. 1987;12(4):362-367. doi:10.1097/00007632-198705000-00010

12. Eisenstein $\mathrm{S}$. The morphometry and pathological anatomy of the lumbar spine in South African negroes and caucasoids with specific reference to spinal stenosis. J Bone Joint Surg Br. 1977;59-B(2):173-180. doi:10.1302/0301-620X.59B2.873978

13. Bajwa NS, Toy JO, Ahn NU. Application of a correlation between the lumbar Torg ratio and the area of the spinal canal to predict lumbar stenosis: a study of 420 postmortem subjects. $J$ Orthop Traumatol. 2013;14(3):207-212. doi:10.1007/s10195-0130237-z

14. Masharawi Y. Lumbar shape characterization of the neural arch and vertebral body in spondylolysis: A comparative skeletal study. Clin Anat. 2012;25(2):224-230. doi:10.1002/ca.21203

15. Krag MH, Weaver DL, Beynnon BD, Haugh LD. Morphometry of the thoracic and lumbar spine related to transpedicular screw placement for surgical spinal fixation. Spine. 1988;13(1):27-32. doi:10.1097/00007632-198801000-00007

16. Marchesi D, Schneider E, Glauser P, Aebi M. Morphometric analysis of the thoracolumbar and lumbar pedicles, anatomo-radiologic study. Surg Radiol Anat. 1988;10(4):317-322. doi:10.1007/BF02107905

17. Ullrich CG, Binet EF, Sanecki MG, Kieffer SA. Quantitative assessment of the lumbar spinal canal by computed tomography. Radiology. 1980;134(1):137-143. doi:10.1148/radiology.134.1.7350593

18. Statistics New Zealand. 2013 Census QuickStats about National Highlights. 2013. www.stats.govt.nz

19. Karantanas AH, Zibis AH, Papaliaga M, Georgiou E, Rousogiannis S. Dimensions of the lumbar spinal canal: variations and correlations with somatometric parameters using CT. Eur Radiol. 1998;8(9):1581-1585. doi:10.1007/s003300050590

20. Gülek B, Durgun B, ??zer HTE, Nazan Alparslan Z, Sarpel T, Erken E. CT-based morphometric dMorphometric Data of L3-L5 vertebrae: anatomic and surgical appVertebrae: Anatomic and Surgical Approach. Neurosurg Q. 2007;17(2):92-97. DOI: doi:\%20 10.1097/WNQ.0b013e318032e0ca

21. Tacar O, Demirant A, Nas K, Altindağ O. Morphology of the lumbar spinal canal in normal adult Turks. Yonsei Med J. 2003;44(4):679-685. doi:10.3349/ymj.2003.44.4.679 
22. Varol T, Iyem C, Cezayirli E, Erturk M, Kayalioglu G, Hayretdag C. Comparative morphometry of the lower lumbar vertebrae: osteometry in dry bones and computed tomography images of patients with and without low back pain. J Int Med Res. 2006;34(3):316-330. doi:10.1177/147323000603400312

23. Zhou SH, McCarthy ID, McGregor AH, Coombs RRH, Hughes SPF. Geometrical dimensions of the lower lumbar vertebrae--analysis of data from digitised CT images. Eur Spine J. 2000;9(3):242-248. doi:10.1007/s005860000140

24. Athiviraham A, Yen D, Scott C, Soboleski D. Clinical correlation of radiological spinal stenosis after standardization for vertebral body size. Clin Radiol. 2007;62(8):776-780. doi:10.1016/j. crad.2007.02.011

25. Lee SY, Kim TH, Oh JK, Lee SJ, Park MS. Lumbar stenosis: a recent update by review of literature. Asian Spine J. 2015;9(5):818-828. doi:10.4184/asj.2015.9.5.818

26. Kitab S, Habboub G, Abdulkareem SB, Alimidhatti MB, Benzel E. Redefining lumbar spinal stenosis as a developmental syndrome: does age matter? J Neurosurg Spine. 2019;31(3):357-365. doi:10.3171/2019.2.SPINE181383

27. Pavlov H, Torg JS, Robie B, Jahre C. Cervical spinal stenosis: determination with vertebral body ratio method. Radiology. 1987;164(3):771-775. doi:10.1148/radiology.164.3.3615879

28. Robertson PA, Nicholson OR. ACC and back injuries: the relevance of pre-existing asymptomatic conditions revisited. $N \mathrm{Z}$ Med J. 2011;124(1335):65-72

29. Blackley HR, Plank LD, Robertson PA. Determining the sagittal dimensions of the canal of the cervical spine. The reliability of ratios of anatomical measurements. J Bone Joint Surg Br. 1999;81(1):110-112. doi:10.1302/0301-620x.81b1.9001
Funding: W.H.C. was the recipient of a NuVasive studentship grant. J.F.B. receives fellowship funding from Medronic and research funding from NuVasive and Smith \& Nephew.

Declaration of Conflicting Interests: The authors have no conflicts of interest to declare relevant to this research.

Ethics Approval: Local ethical body approval was obtained for this study (HDEC 19/NTB/130).

Data Availability Statement: The data that support the findings of this study are available from the corresponding author upon reasonable request.

Corresponding Author: William H. Cook, Department of Surgery, Department of Faculty of Medical and Health Sciences, University of Auckland, Auckland, New Zealand; williamhcook@gmail.com

Published 24 January 2022

This manuscript is generously published free of charge by ISASS, the International Society for the Advancement of Spine Surgery. Copyright (C) 2022 ISASS. To see more or order reprints or permissions, see http:// ijssurgery.com. 